\title{
How successful is successful? Aortic arch shape after successful aortic coarctation repair correlates with left ventricular function
}

\author{
Jan L. Bruse, MSc, ${ }^{a}$ Abbas Khushnood, MD, ${ }^{a}$ Kristin McLeod, PhD, ${ }^{\text {bc }}$ Giovanni Biglino, PhD, ${ }^{a}$ \\ Maxime Sermesant, $\mathrm{PhD},{ }^{\mathrm{c}}$ Xavier Pennec, $\mathrm{PhD},{ }^{\mathrm{c}}$ Andrew M. Taylor, MD, ${ }^{\mathrm{a}}$ Tain-Yen Hsia, MD, ${ }^{\mathrm{a}}$ and \\ Silvia Schievano, $\mathrm{PhD},{ }^{a}$ for the Modeling of Congenital Hearts Alliance Collaborative Group*
}

\begin{abstract}
Objectives: Even after successful aortic coarctation repair, there remains a significant incidence of late systemic hypertension and other morbidities. Independently of residual obstruction, aortic arch morphology alone may affect cardiac function and outcome. We sought to uncover the relationship of arch 3-dimensional shape features with functional data obtained from cardiac magnetic resonance scans.
\end{abstract}

Methods: Three-dimensional aortic arch shape models of 53 patients (mean age, $22.3 \pm 5.6$ years) 12 to 38 years after aortic coarctation repair were reconstructed from cardiac magnetic resonance data. A novel validated statistical shape analysis method computed a 3-dimensional mean anatomic shape of all aortic arches and calculated deformation vectors of the mean shape toward each patient's arch anatomy. From these deformations, 3-dimensional shape features most related to left ventricular ejection fraction, indexed left ventricular end-diastolic volume, indexed left ventricular mass, and resting systolic blood pressure were extracted from the deformation vectors via partial least-squares regression.

Results: Distinct arch shape features correlated significantly with left ventricular ejection fraction $(r=0.42, P=.024)$, indexed left ventricular end-diastolic volume $(r=0.65, P<.001)$, and indexed left ventricular mass $(r=0.44$, $P=.014)$. Lower left ventricular ejection fraction, larger indexed left ventricular end-diastolic volume, and increased indexed left ventricular mass were identified with an aortic arch shape that has an elongated ascending aorta with a high arch height-to-width ratio, a relatively short proximal transverse arch, and a relatively dilated descending aorta. High blood pressure seemed to be linked to gothic arch shape features, but this did not achieve statistical significance.

Conclusions: Independently of hemodynamically important arch obstruction or residual aortic coarctation, specific aortic arch shape features late after successful

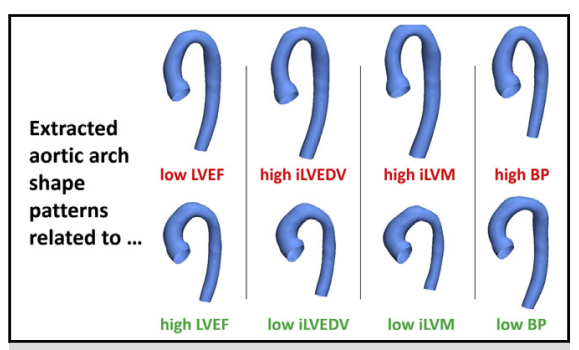

SSM extracted aortic arch shape features related to cardiac function.

\section{Central Message}

Worse left ventricular function and increased left ventricular volume and mass are associated with unique aortic arch shape features late after coarctation repair.

\section{Perspective}

Despite successful aortic coarctation repair, 3D SSM analysis revealed associations of abnormal arch geometry with lower LVEF, increased left ventricular volume, and higher left ventricular mass. 3D morphologic shape features may provide a tool for risk stratification in patients after aortic coarctation repair.

See Editorial Commentary page 428.

See Editorial page 415.

\footnotetext{
From the ${ }^{\mathrm{a} C e n t r e}$ for Cardiovascular Imaging, University College London, Institute of Cardiovascular Science \& Cardiorespiratory Unit, Great Ormond Street Hospital for Children, London, United Kingdom; 'bimula Research Laboratory, Cardiac Modeling Department, Oslo, Norway; and ${ }^{\mathrm{c} I n r i a, ~ A s c l e p i o s ~ T e a m, ~ S o p h i a ~ A n t i p-~}$ olis, France.

This work received funding from the Leducq Foundation (France; grant 09CVD04), FP7 integrated project MD-Paedigree (European Commission), and National Institute for Health Research (United Kingdom; grant No. PDF-2012-05-430). This report incorporates independent research from the National Institute for Health Research Biomedical Research Centre Funding Scheme. The views expressed in this publication are those of the author(s) and not necessarily those of the National Health Service, National Institute for Health Research, or Department of Health. Read at the 96th Annual Meeting of The American Association for Thoracic Surgery, May 14-18, 2016, Baltimore, Maryland.

* Modeling of Congenital Hearts Alliance Collaborative Group: Andrew M. Taylor, Sachin Khambadkone, Silvia Schievano, Marc de Leval, Tain-Yen Hsia
}

(University College London, London, United Kingdom); Edward Bove, Adam Dorfman (University of Michigan, Ann Arbor, Michigan); G. Hamilton Baker, Anthony Hlavacek (Medical University of South Carolina, Charleston, South Carolina); Francesco Migliavacca, Giancarlo Pennati, Gabriele Dubini (Politecnico di Milano, Milan, Italy); Alison Marsden (University of California); Irene Vignon-Clementel (INRIA, Sophia Antipolis, France); and Richard Figliola (Clemson University, Clemson, South Carolina)

Received for publication May 26, 2016; revisions received July 14, 2016; accepted for publication Sept 7, 2016; available ahead of print Oct 21, 2016.

Address for reprints: Tain-Yen Hsia, MD, Cardiac Unit, Great Ormond Street Hospital for Children, NHS Trust, London WC1N 3JH, United Kingdom (E-mail: hsiat@gosh.nhs.uk).

$0022-5223 / \$ 36.00$

Copyright (c) 2016 by The American Association for Thoracic Surgery

http://dx.doi.org/10.1016/j.jtcvs.2016.09.018 

Abbreviations and Acronyms
$\mathrm{BP}=$ blood pressure
BSA = body surface area
CMR = cardiovascular magnetic resonance
CoA $=$ coarctation of the aorta
HLHS = hypoplastic left heart syndrome
iLVEDV $=$ indexed left ventricular end-diastolic volume
iLVM = indexed left ventricular mass
LVEF = left ventricular ejection fraction
PLS $=$ partial least-squares
SSM = statistical shape modeling
2D $\quad=2$-dimensional
$3 \mathrm{D}=3$-dimensional

Scanning this QR code will take you to a video for the article.

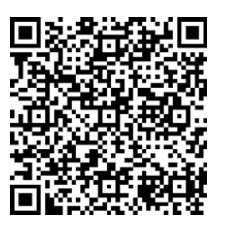

aortic coarctation repair seem to be associated with worse left ventricular function. Analyzing 3-dimensional shape information via statistical shape modeling can be an adjunct to long-term risk assessment in patients after aortic coarctation repair. (J Thorac Cardiovasc Surg 2017;153:418-27)

Despite being perceived as a straightforward lesion with proven and reproducible corrective surgical and interventional techniques, coarctation of the aorta ( $\mathrm{CoA})$ remains a clinical challenge because of a well-recognized high incidence of late complications and morbidities, even after successful repair. ${ }^{1-4}$ In late follow-up, multiple studies have demonstrated a persistence of chronic difficult-to-treat systemic hypertension with associated left ventricular hypertrophy, reduced exercise capacity, and progressive diastolic heart failure. ${ }^{3-6}$ Therefore, long after a "successful" isolated CoA repair with no residual anatomic or hemodynamic obstruction, a significant portion of these patients do not have a "successful" cardiovascular life, requiring lifelong monitoring and chronic pharmacologic management.

As part of the efforts to delineate contributing factors to the CoA puzzle, several investigators have examined the role of aortic arch shape. Discounting the obvious negative effects of residual stenosis or hypoplasia, certain morphologies, or appearance, of the surgically reconstructed aortic arch after isolated CoA repair have been identified to be associated with worse clinical outcome. ${ }^{7-13}$ For example, the much ascribed "gothic" aortic arch with its exaggerated height-to-width ratio and distinct angulation at the crest is likely less desirable than a more rounded and smoother "Romanesque" arch. Despite appearing logical and obvious, a conclusive association between systemic hypertension and gothic arch shape remained elusive, with the additional confounding issues of transverse arch and isthmus sizes adding to the controversy. $^{10,14}$ A large part of these discrepant observations are likely because the majority of these studies applied traditional shape analysis based on linear 2-dimensional (2D) measurements. Being widely variable in shape, angles, and size in 3-dimensional (3D) measurements, surgically reconstructed aortic arches after $\mathrm{CoA}$ repair cannot be adequately analyzed by traditional morphometric methods using a ruler to measure lengths and diameters, because these are insufficient to provide a comprehensive description of the multitude of morphologic permutations. Indeed, even for a "gothic" arch, to fully capture all its nuances and characteristics, a sophisticated approach that quantitatively combines all complex features in 3D is needed. Therefore, we applied a novel, validated 3D statistical shape modeling (SSM) that quantitatively evaluates the ascending aorta/arch morphology as a single, contiguous 3D unit, without the need for manually measuring its numerous dimensions. ${ }^{15-19}$ We hypothesized that unique 3D arch shape features extracted via the SSM are associated with left ventricular functional parameters and systemic blood pressure (BP) in patients late after isolated CoA repair.

\section{PATIENTS AND METHODS \\ Patient Population and Imaging}

We analyzed routine follow-up cardiovascular magnetic resonance (CMR) imaging data (1.5T Avanto MR scanner, Siemens Medical Solutions, Erlangen, Germany) of 53 asymptomatic patients late after isolated CoA (mean age, $22.3 \pm 5.6$ years) (Table 1), including scans from 2007 to 2015 (Figure 1, left). The CMR scans were obtained 12 to 38 years (mean, $20.6 \pm 5.0$ years) after the initial CoA repair, and none had hemodynamically significant residual aortic arch obstruction or CoA requiring revision or reintervention as determined by Doppler echocardiographic interrogation. A total of 36 patients received initial repair during the first year of life $(68 \%), 7$ patients received initial repair in the second year of life, and 10 patients received initial repair more than 5 years after birth (with the oldest age at repair at 10 years). Patients with additional left-sided obstructive lesion (including hypoplastic left heart syndrome [HLHS]) or hypoplastic aortic arch/interrupted aortic arch were excluded, as well as those with aneurysmal dilatation or imaging artifacts due to stents or valve prosthesis. Approximately $80 \%$ of the cohort had an end-to-end CoA repair, whereas approximately half had a bicuspid aortic valve (Table 1). Ethical approval was obtained for the use of image data for research, and all patients or legal guardians gave informed consent.

Left ventricular ejection fraction (LVEF), left ventricular end-diastolic volume, and ventricular mass were calculated from the CMR short-axis 
TABLE 1. Overview of patients' characteristics

\begin{tabular}{lc}
\hline \multicolumn{1}{c}{ Variables } & Mean \pm SD (range) \\
\hline No. of patients & 53 \\
Age at time of CMR (y) & $22.3 \pm 5.6(15.1-38.1)$ \\
Height $(\mathrm{cm})$ & $170.5 \pm 9.5(147-188)$ \\
BSA $\left(\mathrm{m}^{2}\right)$ & $1.83 \pm 0.21(1.44-2.22)$ \\
Aortic valve morphology (TAV/BAV/fBAV) & $(21 / 26 / 6)$ \\
Type of initial repair & $(42 / 1 / 6 / 3 / 1)$ \\
$\quad(\mathrm{E}-\mathrm{E} / \mathrm{ExtE}-\mathrm{E} / \mathrm{flap} / \mathrm{patch} / \mathrm{balloon})$ & \\
LVEF $(\%)$ & $64.1 \pm 7.3(52-78)$ \\
iLVEDV (mL/m $\left.{ }^{2}\right)$ & $78.5 \pm 14.6(57-108)$ \\
iLVM (g/m $\left.{ }^{2}\right)$ & $64.1 \pm 14.7(37-94)$ \\
Systolic resting BP (mm Hg) & $130.0 \pm 17.1(92-163)$ \\
\hline Lower case $i$ indicates parameters indexed to patient BSA. $S D$, Standard deviation; \\
$C M R$, cardiac magnetic resonance; $B S A$, body surface area; $T A V$, tricuspid aortic \\
valve; $B A V$, bicuspid aortic valve; $f B A V$, functionally bicuspid aortic valve; \\
$E-E$, end-to-end anastomosis; $E x t E-E$, extended end-to-end anastomosis; $L V E F$, left \\
ventricular ejection fraction; $i L V E D V$, indexed left ventricular end-diastolic volume; \\
$i L V M$, indexed left ventricular mass; $B P$, blood pressure.
\end{tabular}

stack (Table 1). Resting systolic BP was measured during CMR acquisition using a cuff in the right arm. Body surface area (BSA) was calculated using the Haycock formula, ${ }^{20}$ and parameters were indexed with BSA, where appropriate, denoted with a preceding lower case i (ie, indexed left ventricular end-diastolic volume [iLVEDV] and indexed left ventricular mass [iLVM]).

Aortic arch volumes were segmented and reconstructed from the CMR using a 3D balanced, steady-state free precession whole-heart sequence during mid-diastole rest using Active Contours segmentation tools. ${ }^{21}$ The 3D reconstructed surface models were exported as computational surface meshes and cut consistently with a plane below the aortic root (subannular) and at the level of the diaphragm using the Vascular Modeling Toolkit. ${ }^{22}$ Head and neck vessels and coronary arteries were removed. Before 3D shape analysis, the obtained aortic arch shapes from all patients were prealigned on top of each other using an iterative closest point algorithm in the Vascular Modeling Toolkit. ${ }^{23}$ The meshed, cut, and aligned 3D arch surface models of all 53 aortic arches constituted the input for the SSM (Figure 1, left). ${ }^{15}$

\section{Statistical Shape Analysis Method}

The SSM approach was used to process and analyze all 3D shape information provided by the 53 aortic arch surface models in an integrated computational model, with no need for additional manual measurements or landmarking. ${ }^{24,25}$ Essentially, from the 53 meshes derived from the CMR, the SSM framework (Deformetrica, www.deformetrica.org) computes a template or atlas, that is, the 3D anatomic mean shape (Figure 1, right, blue $).{ }^{18}$ From this template, each patient's aortic arch shape can be fully described by its unique, patient-specific set of deformation vectors ("forward approach") ${ }^{26}$ that recreate each of the 53 patient arches by deforming the template aorta toward the patient shape. All sets of deformation vectors together numerically describe the 3D shape features present in the population, with no need for collection of 2D measurements, coordinates, angles, points, or landmarks, thus allowing statistical analysis to assess how shape variability relates to clinical parameters. ${ }^{15,16}$

Partial least-squares (PLS) regression was applied to the computed deformation vectors to extract 3D shape features (ie, shape deformations) most correlated to the 4 clinical response parameters (LVEF, iLVEDV, iLVM, and BP). ${ }^{15,19,27}$ Before extracting shape features related to functional parameters, size effects due to differences in BSA between patients were removed via a first PLS regression, as described previously. ${ }^{15,19}$ Each extracted shape feature can be visualized in $3 \mathrm{D}^{28}$ by deforming the computed template shape along the extracted deformation vectors ("PLS modes") from low ( -2 standard deviation) to high (+2 standard deviation) values of the response parameter relative to the template (Video 1). Furthermore, a shape vector is calculated, which numerically quantifies how much of the extracted shape features related to the clinical parameter are contained within each patient's arch. ${ }^{15,27}$ Therefore, each patient's 3D shape information, initially provided as a multitude of deformation vectors, is broken down to 1 unitless number that represents the severity of the extracted shape feature within each of the 53 patients in relation to a functional clinical parameter. ${ }^{15,16,19,27}$

The SSM template shape and patient-specific deformation vectors were computed. The template shape was validated as the representative mean shape of the 53-patient cohort in 2 ways. First, geometrically, the gross

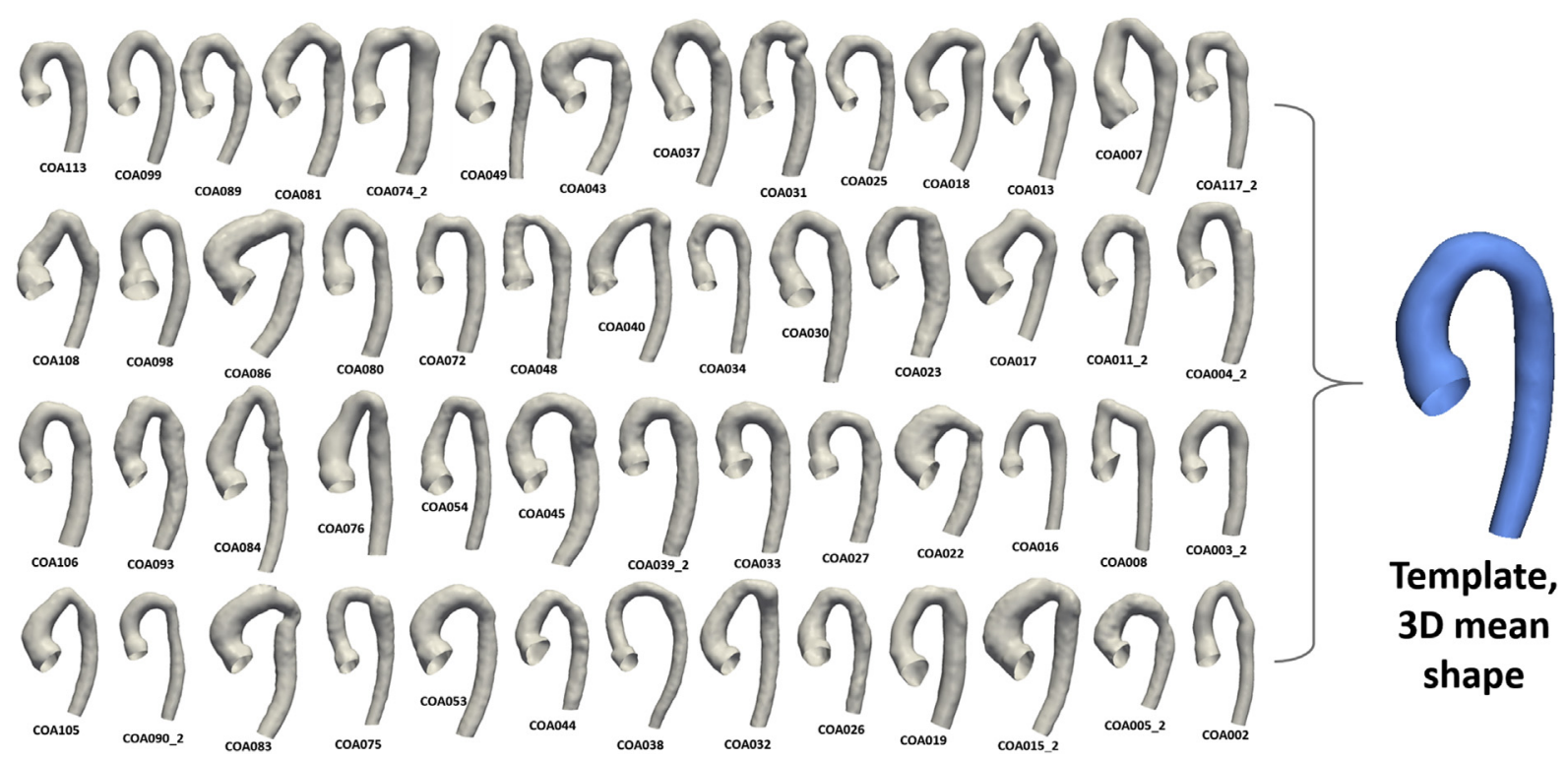

FIGURE 1. Reconstructed 3D surface models of 53 aortic arches after coarctation repair included in this study (grey, left) and computed mean anatomic reference shape based on the input shape population (template shape, blue, right). CoA, Coarctation of the aorta; 3D, 3-dimensional. 


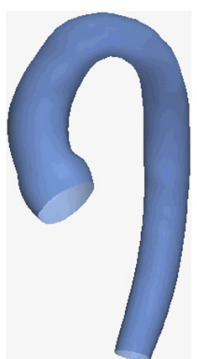

VIDEO 1. Video showing the deformation of the computed template aorta (overlaid in grey) along the derived PLS shape mode for iLVEDV from -2 standard deviation to +2 standard deviation, thus visualizing the $3 \mathrm{D}$ aortic arch shape features most associated with low and high iLVEDV, respectively. Video available at: http://www.jtcvsonline.org/article/ S0022-5223(16)31136-9/addons.

geometric characteristics (volume $\mathrm{V}$, surface area $\mathrm{A}_{\text {surf }}$, and centerline length $\mathrm{L}_{\mathrm{CL}}$ ) of the template were compared with the respective mean values from the entire population extracted via the Vascular Modeling Toolkit. ${ }^{15}$ Second, the template shape was validated numerically via 10 -fold cross-validation: The dataset was divided randomly into 10 subsets, and the template was recomputed 10 times on the basis of a reduced dataset of 9 subsets, until each of the 10 subsets had been left out once to verify independence of the included subjects. ${ }^{27}$

\section{Traditional 3-Dimensional Morphometrics}

To allow for an additional quantitative shape assessment of the derived shape patterns related to functional parameters, we measured traditional morphometric parameters on the computed 3D shapes and the obtained template aorta (Mimics, Materialise, Leuven, Belgium): arch height $\mathrm{h}$ to width $\mathrm{w}$ ratio $(\mathrm{h} / \mathrm{w})$ just above the aortic root and, at the same level, the best fitting ascending and descending aortic diameter $\left(D_{\text {asc }}\right.$ and $D_{\text {desc }}$ respectively) ratio $\left(\mathrm{D}_{\mathrm{asc}} / \mathrm{D}_{\mathrm{desc}}\right)$.

\section{Statistical Analysis}

Associations between the 4 functional parameters (LVEF, iLVEDV, iLVM, and BP) and the shape vectors describing 3D arch shape features were assessed via standard bivariate correlation analyses. Pearson's $r$ is reported for parametric, normally distributed data. Non-normality was assumed if the Shapiro-Wilk test was significant, assuming a significance level of $P$ less than .05. For correlation analyses, computed $P$ values were adjusted for multiple comparisons via permutation tests with 100,000 permutations at an $\alpha$-level of $0.05 .{ }^{29}$ Because PLS regression is sensitive to outliers, ${ }^{30}$ the Cook's distance (measuring the influence of a single subject on the final regression results) was computed for each PLS regression run. For all the PLS regression runs using functional parameters, 2 subjects exceeding 4 times the mean Cook's distance were considered to be influential and subsequently removed from the respective shape feature extraction. Before extracting shape features related to functional parameters, size effects were removed by regressing the computed deformation vectors with BSA. One subject had to be removed from subsequent analyses for being influential to the regression, following the Cook's distance analysis. Statistical tests were performed in MATLAB (MathWorks, Natick, Mass) and SPSS (IBM SPSS Statistics, SPSS Inc, Chicago, Ill).

\section{RESULTS}

\section{Template Aortic Arch}

Qualitatively, the template aorta, derived as the mean 3D aorta shape computed from the 53-patient cohort, had a moderately increased height-to-width ratio and a nonangulated Romanesque-type arch shape with no distinct narrowing or recoarctation (Figure 1). These features were typical of what a surgeon or cardiologist would label as a "perfect" aortic arch after CoA repair. As a validation, traditional morphometric parameters measured on the template shape were close to their respective mean values as calculated from the entire cohort (Table 2), with an overall deviation of $3.3 \%$ (individual deviations $\Delta \mathrm{V}=5.6 \%, \Delta \mathrm{A}_{\text {surf }}=3.0 \%$, and $\left.\Delta \mathrm{L}_{\mathrm{CL}}=1.4 \%\right)$. In addition, cross-validation confirmed that removing subjects randomly from the population did not change the template shape significantly (average surface distance between original template shape and cross-validated shapes $\Delta \mathrm{D}_{\text {surf }}=0.285 \pm 0.07 \mathrm{~mm}$ ). The template was validated as a representative anatomic mean shape of our cohort.

\section{Correlations of Arch Shape Features With Left Ventricular Function, Volume, and Mass}

PLS regression results showed that derived 3D shape vectors were significantly correlated with LVEF, even after adjusting for multiple comparisons $(r=0.42, P=.024)$. Shape features that were associated with lower LVEF

TABLE 2. Morphometric parameters measured on the computed 3-dimensional shapes and respective population averages

\begin{tabular}{|c|c|c|c|c|c|c|c|}
\hline 3D shape & $\mathrm{V}\left(\mathrm{mm}^{\mathbf{3}}\right)$ & $A_{\text {surf }}\left(\mathbf{m m}^{2}\right)$ & $\mathbf{L}_{\mathbf{C L}}(\mathbf{m m})$ & $\mathbf{L}_{\mathbf{T o}}$ & $\mathbf{D}_{\mathbf{a v}}(\mathbf{m m})$ & $\mathbf{D}_{\text {asc }} / \mathbf{D}_{\text {desc }}$ & $\mathbf{h} / \mathbf{w}$ \\
\hline Low LVEF shape & 97,804 & 18,408 & 253.65 & 1.85 & 20.90 & 1.11 & 1.33 \\
\hline High LVEF shape & 75,583 & 14,598 & 207.85 & 1.64 & 19.62 & 1.50 & 0.93 \\
\hline Low iLVEDV shape & 72,193 & 13,607 & 190.48 & 1.52 & 19.71 & 1.73 & 0.73 \\
\hline High iLVEDV shape & 106,257 & 19,824 & 268.62 & 1.95 & 20.78 & 1.08 & 1.43 \\
\hline Low iLVM shape & 69,599 & 13,042 & 183.12 & 1.66 & 19.25 & 1.96 & 0.70 \\
\hline High iLVM shape & 117,210 & 21,145 & 276.04 & 1.81 & 21.32 & 0.96 & 1.47 \\
\hline Low BP shape & 87,759 & 16,873 & 234.81 & 1.64 & 20.48 & 1.15 & 0.97 \\
\hline High BP shape & 85,570 & 16,177 & 228.24 & 1.87 & 20.05 & 1.41 & 1.29 \\
\hline Template & 88,108 & 16,665 & 230.84 & 1.74 & 20.56 & 1.32 & 0.94 \\
\hline Population average & 93,111 & 17,166 & 233.96 & 1.80 & 19.40 & - & - \\
\hline
\end{tabular}

3D, 3-dimensional; $V$, volume; $A_{\text {surf }}$, arch surface area; $L_{C L}$, centerline length; $L_{T o}$, centerline tortuosity; $D_{a v}$, average diameter along the centerline; $D_{a s d} / D_{\text {desc }}$, ascending to descending diameter ratio; $h / w$, arch height to width ratio; $L V E F$, left ventricular ejection fraction; $i L V E D V$, indexed left ventricular end-diastolic volume; $i L V M$, indexed left ventricular mass; $B P$, blood pressure. 

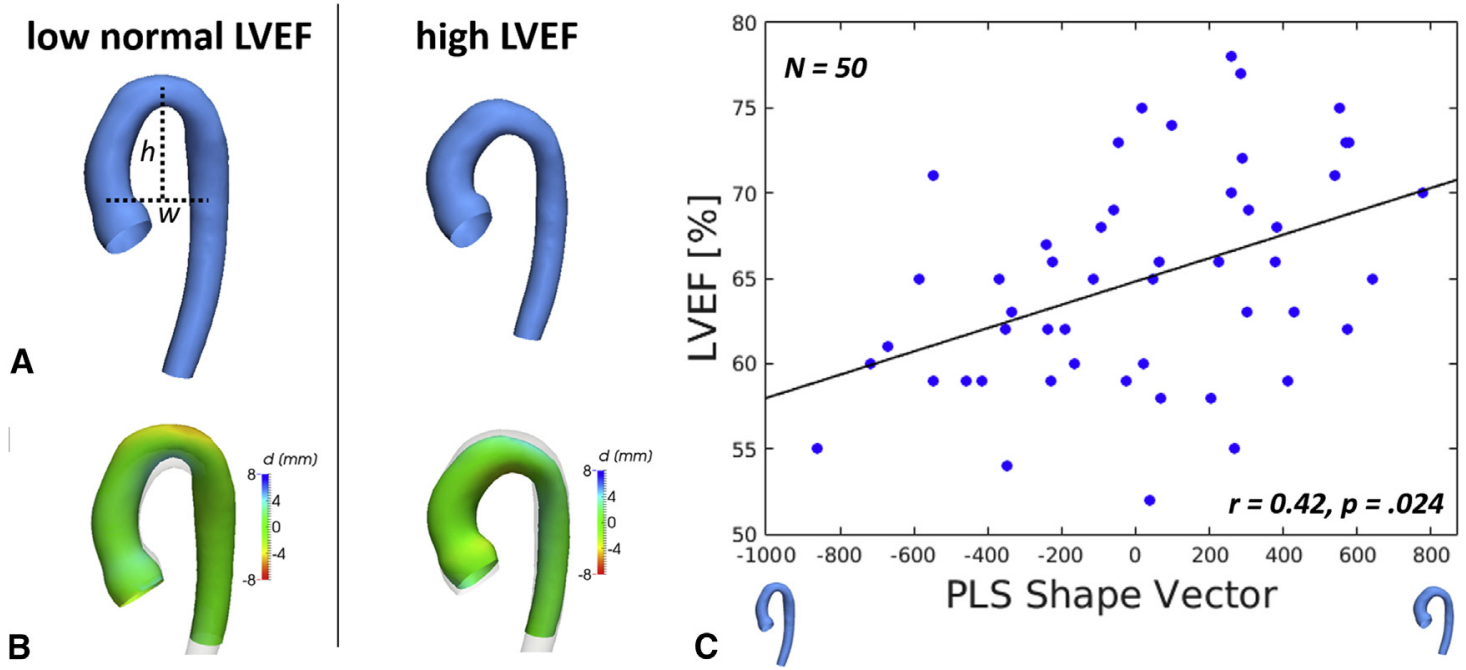

FIGURE 2. Visualization of 3D aortic arch shape patterns associated with LVEF, deforming the template shape from low ( -2 standard deviation) to high ( +2 standard deviation) values of the response parameter LVEF (computed shape features visualized in blue) and definition of height to width ratio h/w (A). Color maps show local 3D shape deviations as the distance in millimeters between the computed shapes and the template shape, overlaid in grey; blue colors relate to inward deformations; red colors relate to outward deformations from the template (B). Standard bivariate correlation analysis was used to evaluate numerically how strongly the found patterns were related to LVEF (C). Low (normal) LVEF thereby was associated with an overall large arch with high $\mathrm{h} / \mathrm{w}$ ratio and a slim ascending and mildly hypoplastic transverse arch, whereas high LVEF related to more rounded and compact arches. $L V E F$, Left ventricular ejection fraction; $h / w$, arch height to width ratio; $P L S$, partial least-squares.

include an overall gothic-like aortic arch shape with elevated height-to-width ratio $(\mathrm{h} / \mathrm{w}=1.33$, Table 2$)$ and an elongated ascending and shorter transverse arch and a slight size mismatch between a smaller isthmus and a larger descending aorta. In contrast, a shorter, generally more rounded arch was associated with higher LVEF $(\mathrm{h} / \mathrm{w}=0.93)$ (Figure 2). Moreover, the nearly identical aortic arch features associated with lower LVEF were observed to correlate with both increased iLVEDV $(\mathrm{h} / \mathrm{w}=1.73 ; r=0.65, P<.001)$ (Figure 3$)$ and higher $\operatorname{iLVM}(\mathrm{h} / \mathrm{w}=1.47 ; r=0.44, P=.014)$ (Figure 4).

Conversely, aortic arches associated with both low iLVEDV and low iLVM featured an overall more compact and rounded (Romanesque) arch shape (h/w $=0.73$ and 0.70 , respectively) with a larger ascending arch that tapers into a relatively smaller distal transverse and isthmus arch continuation $\left(\mathrm{D}_{\mathrm{asc}} / \mathrm{D}_{\text {desc }}=1.73\right.$ and 1.96 , respectively).
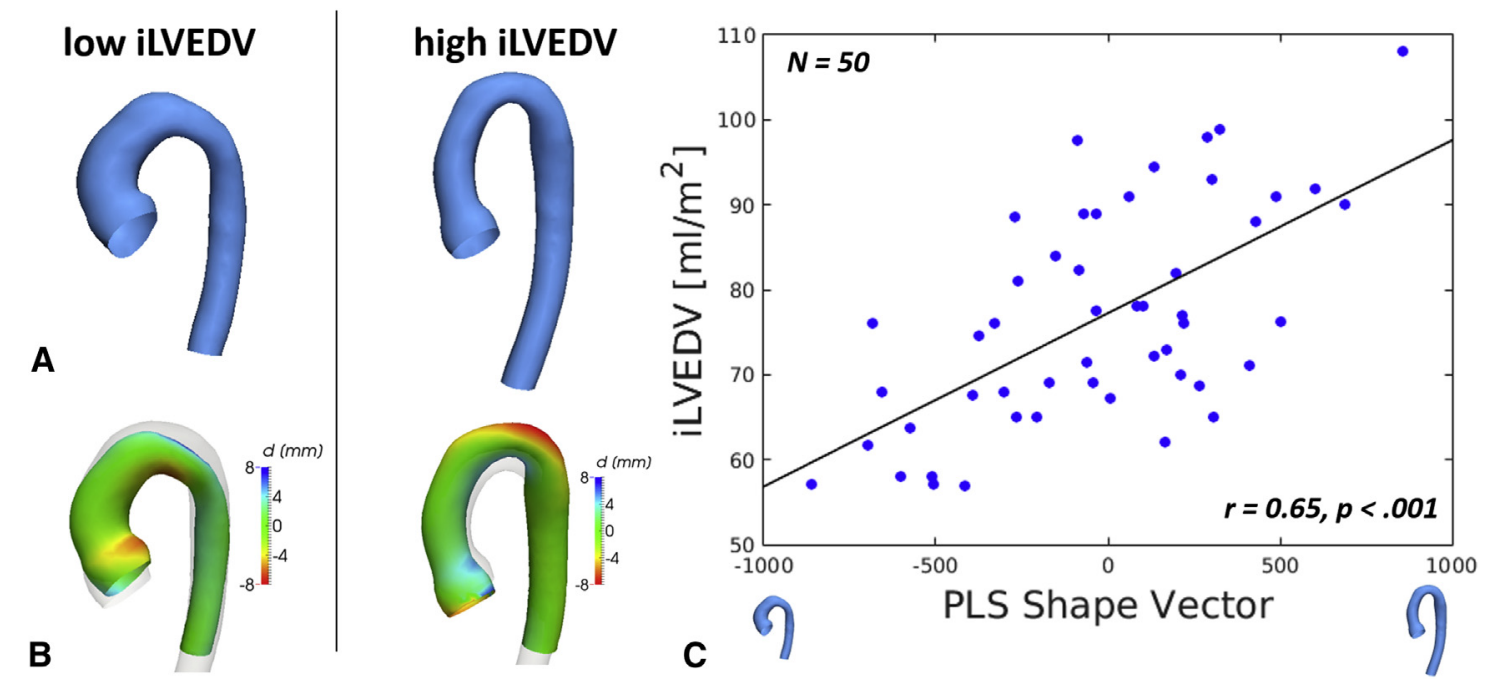

FIGURE 3. Elevated iLVEDV was associated with overall larger and tortuous arches with high $\mathrm{h} / \mathrm{w}$ ratio and a long, slim ascending and proximally hypoplastic transverse aortic arch. Extracted shape patterns are visualized as deformations of the template in blue (A), and local deviations from the template shape are shown as color maps in (B). Bivariate correlation analysis showed strong association between shape vector to iLVEDV (C). iLVEDV, Indexed left ventricular end-diastolic volume; $P L S$, partial least-squares. 

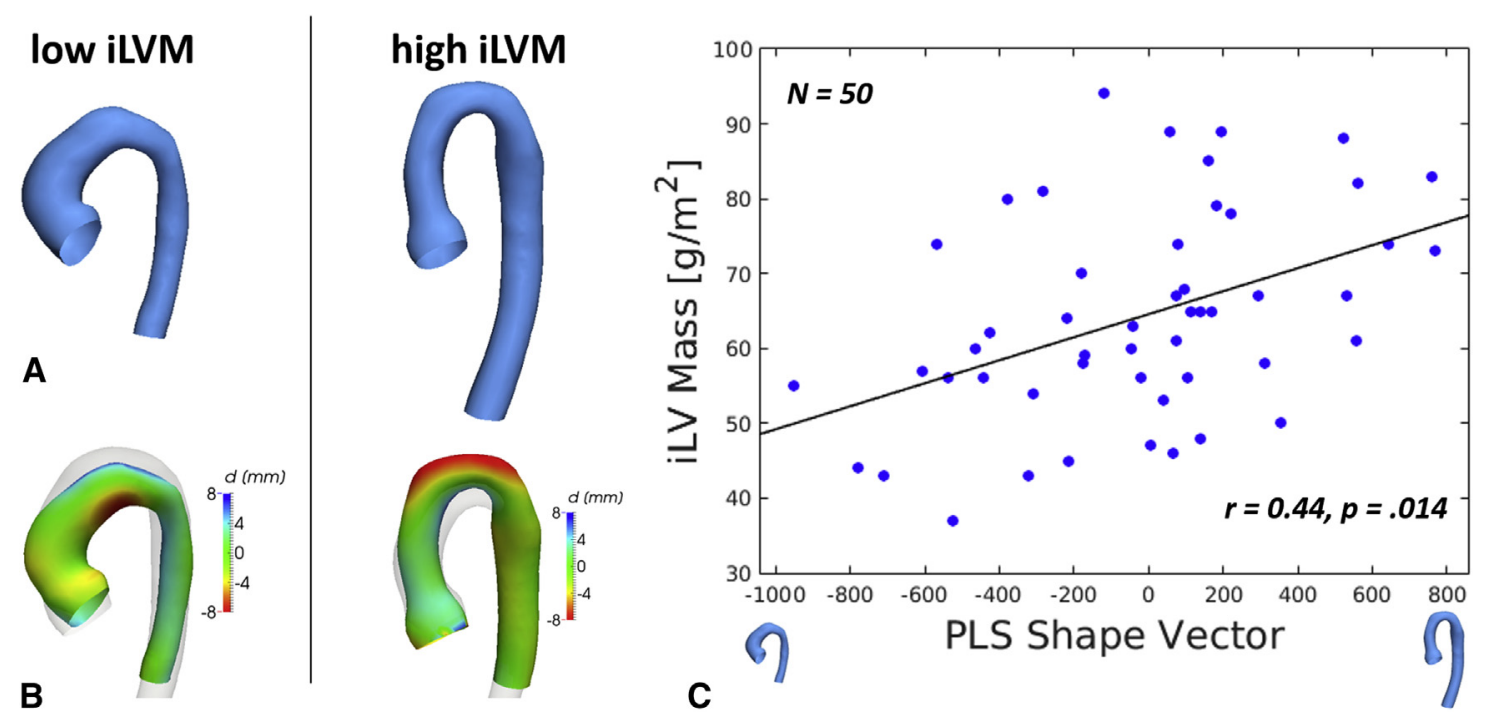

FIGURE 4. Elevated iLVM was associated with an overall large and tortuous, high $\mathrm{h} / \mathrm{w}$ ratio arch shape, showing a very slim ascending and transverse arch with mild narrowing at the isthmus region and a long and dilated descending aorta. Extracted shape patterns are visualized as deformations of the template in blue (A), and local deviations from the template shape are shown as color maps (B). Bivariate correlation analysis showed significant association between aortic arch shape vector to iLV mass (C). $i L V M$, Indexed left ventricular mass; $i L V$, indexed left ventricle; $P L S$, partial least-squares.

\section{Correlations of Arch Shape Features with Systolic Blood Pressure at Rest}

High systolic resting BP was identified with a gothic-type arch shape $(\mathrm{h} / \mathrm{w}=1.41)$ presenting with a mild ascending arch dilation and a narrow and short transverse arch with exaggerated acute angulation at its apex, followed by a mild diameter increase from isthmus to descending aorta (Figure 5). The aortic arch shape associated with low BP showed a more crenel-like, longer, and rounded aortic arch. Although initially significant in stand-alone statistics, this shape to BP association did not reach statistical significance after adjusting for multiple comparisons $(r=0.32, P=.160)$.

\section{DISCUSSION}

The goal of surgical repair of $\mathrm{CoA}$ is to restore unobstructed systemic blood flow through the aortic arch, with the additional beneficial consequence of lifelong freedom from hypertension. However, an observation is emerging that a significant number of patients late after

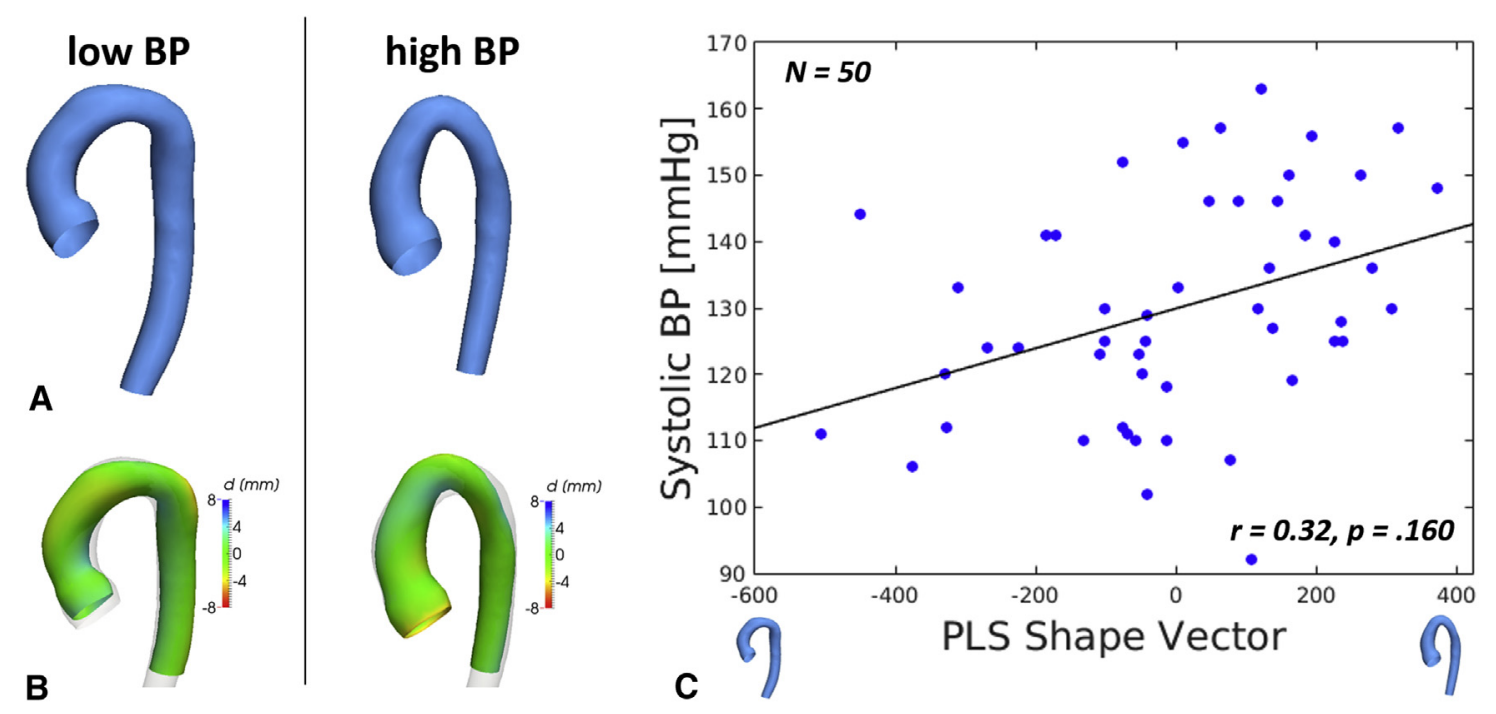

FIGURE 5. High systolic resting BP related to an overall gothic-type and tortuous arch shape with mildly dilated ascending aorta and signs of residual narrowing at the isthmus section, compared with a crenel-like arch for lower BP values. Results were not significant after adjusting for multiple comparisons (C). Extracted shape patterns are visualized as deformations of the template in blue (A), and local deviations from the template shape are shown as color maps (B). BP, Blood pressure; PLS, partial least-squares. 
what appeared to be a successful CoA repair with no residual obstructive lesion experience systemic hypertension and an exaggerated $\mathrm{BP}$ response to exercise. ${ }^{3-6}$ Although intrinsic abnormal aortic wall properties exist in patients with aortic arch anomalies, investigations into the role of arterial elastance and compliance have not yielded a definitive mechanistic link to systemic hypertension in patients after CoA repair. The appearance of the aortic arch in patients after CoA repair recently has been called into question as a potential contributor to poor late outcomes. $^{2}$ Again, traditional linear 2D measurements have led to conflicting results. There is no question that the aortic arches in patients who underwent CoA repair look different than those of healthy individuals. This is confounded by the fact that not only different operative techniques exist but also the entire ascending aorta aortic and arch isthmus descending aorta complex can vary greatly in size and shape from patient to patient, in addition to differing incidences of residual arch obstruction, dilatation, and tortuosity. Therefore, to accurately capture all the features within an aortic arch after CoA repair requires a sophisticated analysis of its modified (ie, repaired) and unmodified (ie, native) characteristics in 3D space.

In this study, by using a novel 3D SSM that is capable of extracting and visualizing complex aortic arch shape features, unique aortic arch features late after CoA repair were found to correlate with poorer left ventricular function and increased left ventricular volume and mass. This methodology, which combines CMR-based computational modeling and advanced statistical analysis, is based on defining a mean aortic arch that is representative of the average shape from a specific patient cohort. Adopting a template aorta based on subjects with normal hearts and normal aortic arches would be meaningless for patients with CoA because of the compulsory aortic arch reconstruction and the known variations in arch geometry among these patients. Therefore, the template aorta (Figure 1) is derived from the 53-patient cohort as the "norm" for a patient with CoA, with a smooth "candy-cane"-like curvature that extends from the ascending aorta to the descending. Free from obvious obstruction or acute changes in size and cross-sectional area, this template typically would be one that surgeons and cardiologists would consider a successful repaired aortic arch.

From this template, the SSM quantified shape features or deformation vectors that correlated with lower LVEF, larger iLVEDV, and higher iLVM. This suggests that independently of hemodynamically important residual obstruction, stenosis, or hypoplasia, how the aortic arch is shaped can be associated with poorer left ventricular performance. It appears that the common features linked with these worse left ventricular functional parameters are aortic arches with an elongated ascending aorta, an increased height-to-width ratio, and a shorter transverse arch and a slight size mismatch between a smaller isthmus and a larger descending aorta. Common features observed in those aortic arches associated with better left ventricular parameters included an overall smaller arch complex, a slightly oversized ascending aorta, a more rounded and longer transverse arch, and a smoother match between isthmus and descending aorta. However, it should be noted that all the patients were asymptomatic of heart failure. Indeed, the lowest LVEF in the cohort was $52 \%$, and the highest iLVM and iLVEDV were within acceptable limits. Nonetheless, the combination of a higher left ventricular mass and volume is known to be a risk marker for increased cardiovascular morbidity, including coronary artery disease and cerebral vascular accidents. , $^{4}$

Although residual arch stenosis has been associated with higher iLVM by Ong and colleagues, ${ }^{7}$ the strong correlation uncovered in this study highlighted that the importance of shape alone, independently of flow obstruction, could play a role in late CoA outcomes. Along the same vein, our study also examined the role of the overall proportion of the intrathoracic aorta. With the aortic arch geometry reconstruction uniformly obtained from the aortic root to the diaphragm in each patient, and influence of different body size eliminated, smaller and more compact, rounded arches seemed to be associated with better left ventricular function. Yet, overall arch size cannot be accounted for when using traditional morphometric. Therefore, the overall intrathoracic aorta size seems to be relevant, further justifying assessing the 3D shape anatomy contiguously in whole.

The trend that elevated resting BP was associated with a gothic-type aortic arch shape was in line with other studies, some of which also showed an association with an exaggerated BP response to exercise. ${ }^{32,33}$ The presence of abnormal wall properties of the entire systemic arterial tree, such as reduced compliance and distensibility, has been shown to exist in patients with CoA with hypertension. ${ }^{5,6,34}$ The present shape analysis methodology cannot account for aortic wall property variations, which could confound the association between arch shape and hypertension. Combined with our recent development of wave-intensity analysis that can evaluate arterial wall distensibility and elastance, it is possible in the future that these 2 CMR-derived methods can reveal a clearer relationship between aortic arch shape and hypertension.

There is a similarity and difference seen between aortic arch shape features in patients with CoA and those in patients after the Norwood procedure for HLHS. In a recent study applying the same methodology in patients with HLHS after the Norwood-type aortic arch reconstruction, ${ }^{16}$ we described a significant correlation between unique aortic arch shape features and increased right ventricular end-diastolic volume and other adverse outcomes. Although these 2 studies concurrently demonstrate the possible importance of aortic arch shape, there is a major difference: The aortic arch shape and morphology after the Norwood 
procedure are potentially modifiable, but those in patients with CoA after successful repair are more difficult to modify. The technique and manner in which the combination of Damus-Kay-Stansel/arch reconstruction is performed at stage 1 Norwood clearly are major determinants of the eventual shape of the aortic arch in patients with HLHS. However, as seen in this study, the deterministic factors in the shape features of an aortic arch late after CoA repair are essentially intrinsic or inherently altered, that is, a gothic or Romanesque aortic arch is born that way. In the absence of obvious hypoplasia or stenosis, one typically would not surgically intervene on a gothic-appearing aortic arch, nor would one reconstruct an arch that we have identified to be associated with worse left ventricular parameters. In fact, in reviewing the computed tomography or magnetic resonance scans of a patient before this study, one likely would have described such an aortic arch to be a "successful" CoA repair.

\section{Study Limitations}

Although our study has shown these previously unknown relationships between aortic shape and clinical parameters in patients after CoA repair, it is important to note that this study does not reveal any mechanistic insight as to why the specific distortion or deformation in some shape features would be important, and thus we cannot provide a causal relationship to our observations. Whether these deranged aortic shapes lead to altered impedance or perturbed aortic outflow is unknown. Further studies, perhaps with 4-dimensional $\mathrm{CMR}^{35}$ and advanced computational fluid dynamics modeling, in which realistic time-dependent and pulsatile flow/pressure characteristics can be simulated and examined, may yield important insights into the flow disturbances that can lead to worse cardiac function and clinical outcomes.

\section{CONCLUSIONS}

In this study, we assessed aortic arch morphology post-CoA repair using a novel SSM approach to extract 3D arch shape features related to functional parameters acquired during routine follow-up magnetic resonance assessment. We found a previously unknown association of unique aortic arch shape with lower LVEF and elevated left ventricular end-diastolic volume and mass. Moreover, our study suggested that a gothic aortic arch might be correlated with hypertension, but this was not conclusive. Nonetheless, this study did confirm that aortic arch shape in patients post-CoA repair could be related to cardiac function, and in so doing it also highlighted that a few isolated 2D morphometric measurements could not fully capture the intricate and complex combination of shape features in an aortic arch. Adaptation of the statistical shape analysis method using extracted 3D aortic arch geometry might provide a predictive tool to risk stratify patients after successful CoA repair for the late development of hypertension and left ventricular functional derangements.

\section{Conflict of Interest Statement}

Authors have nothing to disclose with regard to commercial support.

\section{References}

1. Hauser M. Exercise blood pressure in congenital heart disease and in patients after coarctation repair. Heart. 2003;89:125-6.

2. De Caro E, Trocchio G, Smeraldi A, Calevo MG, Pongiglione G. Aortic arch geometry and exercise-induced hypertension in aortic coarctation. Am J Cardiol. 2007;99:1284-7.

3. Puranik R, Tsang VT, Puranik S, Jones R, Cullen S, Bonhoeffer P, et al. Late magnetic resonance surveillance of repaired coarctation of the aorta. Eur $J$ Cardiothorac Surg. 2009;36:91-5.

4. Brown ML, Burkhart HM, Connolly HM, Dearani JA, Cetta F, Li Z, et al. Coarctation of the aorta: lifelong surveillance is mandatory following surgical repair. J Am Coll Cardiol. 2013;62:1020-5.

5. O'Sullivan J. Late hypertension in patients with repaired aortic coarctation. Curr Hypertens Rep. 2014;16:1-6.

6. Canniffe C, Ou P, Walsh K, Bonnet D, Celermajer D. Hypertension after repair of aortic coarctation-a systematic review. Int J Cardiol. 2013;167:2456-61.

7. Ong CM, Canter CE, Gutierrez FR, Sekarski DR, Goldring DR. Increased stiffness and persistent narrowing of the aorta after successful repair of coarctation of the aorta: relationship to left ventricular mass and blood pressure at rest and with exercise. Am Heart J. 1992;123:1594-600.

8. Vriend JWJ, Zwinderman AH, de Groot E, Kastelein JJP, Bouma BJ, Mulder BJM. Predictive value of mild, residual descending aortic narrowing for blood pressure and vascular damage in patients after repair of aortic coarctation. Eur Heart J. 2005;26:84-90.

9. Weber HS, Cyran SE, Grzeszczak M, Myers JL, Gleason MM, Baylen BG. Discrepancies in aortic growth explain aortic arch gradients during exercise J Am Coll Cardiol. 1993;21:1002-7.

10. Lee MGY, Kowalski R, Galati JC, Cheung MMH, Jones B, Koleff J, et al Twenty-four-hour ambulatory blood pressure monitoring detects a high prevalence of hypertension late after coarctation repair in patients with hypoplastic arches. J Thorac Cardiovasc Surg. 2012;144:1110-8.

11. Ou P, Bonnet D, Auriacombe L, Pedroni E, Balleux F, Sidi D, et al. Late systemic hypertension and aortic arch geometry after successful repair of coarctation of the aorta. Eur Heart J. 2004;25:1853-9.

12. Ou P, Celermajer DS, Mousseaux E, Giron A, Aggoun Y, Szezepanski I, et al Vascular remodeling after "successful" repair of coarctation: impact of aortic arch geometry. J Am Coll Cardiol. 2007;49:883-90.

13. Ou P, Celermajer DS, Raisky O, Jolivet O, Buyens F, Herment A, et al. Angular (Gothic) aortic arch leads to enhanced systolic wave reflection, central aortic stiffness, and increased left ventricular mass late after aortic coarctation repair: evaluation with magnetic resonance flow mapping. J Thorac Cardiovasc Surg. 2008; $135: 62-8$.

14. Ntsinjana HN, Biglino G, Capelli C, Tann O, Giardini A, Derrick G, et al. Aortic arch shape is not associated with hypertensive response to exercise in patient with repaired congenital heart diseases. J Cardiovasc Magn Reson. 2013;15:101.

15. Bruse JL, McLeod K, Biglino G, Ntsinjana HN, Capelli C, Hsia T-Y, et al A statistical shape modelling framework to extract 3D shape biomarkers from medical imaging data: assessing arch morphology of repaired coarctation of the aorta. BMC Med Imaging. 2016;16:40.

16. Bruse JL, Cervi E, McLeod K, Biglino G, Sermesant M, Pennec X, et al. Looks do matter! Aortic arch shape following hypoplastic left heart syndrome palliation correlates with cavopulmonary outcomes. Ann Thorac Surg. September 1, 2016 [Epub ahead of print].

17. Leonardi B, Taylor AM, Mansi T, Voigt I, Sermesant M, Pennec X, et al. Computational modelling of the right ventricle in repaired tetralogy of Fallot: can it provide insight into patient treatment? Eur Heart J Cardiovasc Imaging. 2013;14:381-6.

18. Durrleman S, Prastawa M, Charon N, Korenberg JR, Joshi S, Gerig G, et al Morphometry of anatomical shape complexes with dense deformations and sparse parameters. Neuroimage. 2014;101:35-49.

19. Bruse JL, McLeod K, Biglino G, Ntsinjana HN, Capelli C, Hsia T-Y, et al. A non-parametric statistical shape model for assessment of the surgically repaired aortic arch in coarctation of the aorta: how normal is abnormal? In: Camara O, 
Pop M, Rhode K, Sermesant M, Smith N, Young A, eds. Statistical Atlases and Computational Models of the Heart 2015. Munich: Springer International Publishing Switzerland; 2016:21-9.

20. Haycock GB, Schwartz GJ, Wisotsky DH. Geometric method for measuring body surface area: a height-weight formula validated in infants, children, and adults. J Pediatr. 1978;93:62-6.

21. Yushkevich PA, Piven J, Hazlett HC, Smith RG, Ho S, Gee JC, et al. User-guided $3 \mathrm{D}$ active contour segmentation of anatomical structures: significantly improved efficiency and reliability. Neuroimage. 2006;31:1116-28.

22. Antiga L, Piccinelli M, Botti L, Ene-Iordache B, Remuzzi A, Steinman DA. An image-based modeling framework for patient-specific computational hemodynamics. Med Biol Eng Comput. 2008;46:1097-112.

23. Besl PJ, McKay ND. A method for registration of 3-D shapes. IEEE Trans Pattern Anal Mach Intell. 1992;14:239-56.

24. Young AA, Frangi AF. Computational cardiac atlases: from patient to population and back. Exp Physiol. 2009;94:578-96.

25. Durrleman S, Pennec X, Trouvé A, Ayache N. Statistical models of sets of curves and surfaces based on currents. Med Image Anal. 2009;13:793-808.

26. Durrleman S, Pennec X, Trouvé A, Ayache N. A forward model to build unbiased atlases from curves and surfaces. In: 2nd MICCAI Workshop on Mathematical Foundations of Computational Anatomy, New York, NY, October 2008. Le Chesnay, France: Inria; 2008:68-79.

27. Mansi T, Voigt I, Leonardi B, Pennec X, Durrleman S, Sermesant M, et al. A statistical model for quantification and prediction of cardiac remodelling: application to tetralogy of Fallot. IEEE Trans Med Imaging. 2011;30:1605-16.

28. Ahrens J, Geveci B, Law C. ParaView: an end user tool for large data visualization. In: Johnson CR, Hansen CD, eds. Visualization Handbook. Burlington, MA: Elsevier Butterworth-Heinemann; 2005:717.

29. Groppe DM, Urbach TP, Kutas M. Mass univariate analysis of event-related brain potentials/fields I: a critical tutorial review. Psychophysiology. 2011;48:1711-25.

30. Daszykowski M, Kaczmarek K, Vander Heyden Y, Walczak B. Robust statistics in data analysis-a review: basic concepts. Chemometr Intell Lab Syst. 2007;85:203-19.

31. Levy D, Garrison RJ, Savage DD, Kannel WB, Castelli WP. Prognostic implications of echocardiographically determined left ventricular mass in the Framingham Heart Study. N Engl J Med. 1990;322:1561-6.

32. Donazzan L, Crepaz R, Stuefer J, Stellin G. Abnormalities of aortic arch shape, central aortic flow dynamics, and distensibility predispose to hypertension after successful repair of aortic coarctation. World J Pediatr Congenit Heart Surg. 2014;5:546-53.

33. Ou P, Mousseaux E, Celermajer DS, Pedroni E, Vouhe P, Sidi D, et al. Aortic arch shape deformation after coarctation surgery: effect on blood pressure response. J Thorac Cardiovasc Surg. 2006;132:1105-11.

34. Lombardi KC, Northrup V, McNamara RL, Sugeng L, Weismann CG. Aortic stiffness and left ventricular diastolic function in children following early repair of aortic coarctation. Am J Cardiol. 2013;112:1828-33.

35. Frydrychowicz A, Markl M, Hirtler D, Harloff A, Schlensak C, Geiger J, et al. Aortic hemodynamics in patients with and without repair of aortic coarctation: in vivo analysis by $4 \mathrm{~d}$ flow-sensitive magnetic resonance imaging. Invest Radiol. 2011;46:317-25.

Key Words: coarcation of the aorta, shape analysis, aortic arch

\section{Discussion}

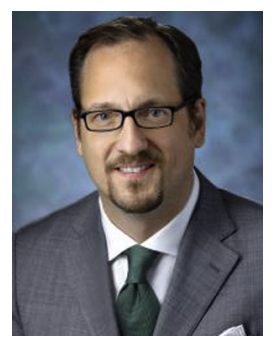

Dr L. Vricella (Baltimore, Md). I congratulate you and the group at Great Ormond Street for an elegant presentation and for attempting to establish the fact that morphology is independently correlated to late outcomes in the absence of residual left ventricular outflow tract obstruction. I think this applies to interrupted aortic arches, subclavian flap aortoplasties, and other repairs that entail some form of change in the morphology of the aortic arch.
I have a comment and then 3 questions for you. The comment has to do with the fact that you established 4 parameters as your surrogates of late outcome. In all fairness, in the article the ejection fraction was $55 \%$ at the lowest, and left ventricular end-diastolic volume and mass were within normal range. To these 3 cardiac parameters, you added BP at baseline.

I looked at the age of your patients, and of 53 patients, 17 were beyond infancy at the time of repair, with 10 actually older than 5 years. How do you correlate older age at the time of repair, which you know is associated with late hypertension at baseline, with the results of your analysis?

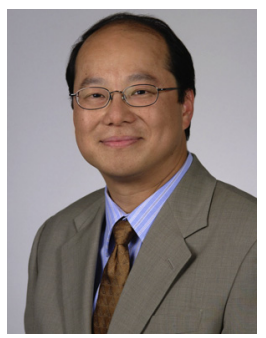

Dr Hsia (London, United Kingdom). In this cohort of 53 patients, the low ejection fraction was just at the borderline of being low/normal. However, if you look at the spread of the left ventricular mass and end-diastolic volume, there were patients who had abnormal ranges in terms of increased left ventricular mass and increased end-diastolic volume.

We cannot account for age as a specific variable. Obviously in this study, there is a wide range of ages at the time of magnetic resonance, but all these patients are late after the initial coarctation repair. So the question that we really tried to answer is, does shape correlate with some of the left ventricular performance parameters later after successful coarctation repair, and I think we've successfully done that.

Dr Vricella. You averaged all your 53 coarctation repairs to establish your template. So with the limitations of ordering magnetic resonance imaging in normal patients, why not compare coarctation repairs with normal individuals?

Dr Hsia. The patients we are studying are not normal. If the goal is to understand how shape or morphology affects late outcomes in patients with a specific disease, what you really want to know is how the patient population is affected by this particular disease process. In other words, using normal subjects to create the template would lead to misinformation because none of the patients after coarctation repair have a normal aortic arch.

Thus, the question we want to know is what kind of deviation, in terms of shape features, within this disease process could be linked to worse later outcomes. By obtaining the template from the cohort of patients after coarctation repair, we arrive at an arch that would be representative of an arch that we typically would be pleased with, and from that, you look at each patient's characteristics and see how that affects the outcome.

Dr Vricella. Do you have a mechanistic explanation for your findings?

Dr Hsia. This study does not provide any mechanistic insight into why those specific aortic arch shapes correlate 
with the parameters that we have set out to examine. What it does is give us an understanding that looks actually do matter.

This goes back to, I think for most people here in the audience, that as surgeons we were taught early on as trainees that if an anastomosis or reconstruction doesn't look good, it is unlikely to work well. I think it goes back to the fact that when something doesn't look quite right, there are some morphologic features independent of hemodynamic pressure gradients or stenosis or hypoplasia that can affect those issues.

To further explore a mechanistic explanation, we are undertaking sophisticated fluid dynamic studies and a 4-dimensional magnetic resonance study to understand the subtle flow characteristics that may point to a mechanistic reason for what we see here today.

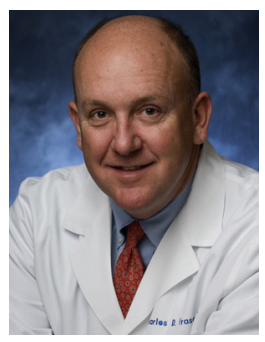

Dr C. Fraser (Houston, Tex). Just as you said, we want to figure out ways to do our work better. Are you applying this work to the efforts our cardiology colleagues are bringing to stenting arches and coarctations? I'm curious about that.
Dr Hsia. A weakness that the shape analysis doesn't address is the aortic wall property issues associated with having stents or foreign patches and how that would confound the discovery here. Another issue is that metallic stents would interfere with or prevent sufficient data acquisition with magnetic resonance imaging.

In terms of making recommendations, we are not at the point to imply anything. One can argue that according to what you see, if you have a patient who has a gothic aortic arch after coarctation repair, perhaps you should surgically intervene to make the arch look better despite not having any hemodynamic residual. We cannot make this suggestion at this point of the investigation.

The important thing to note is that in this particular study, the focus is on isolated aortic coarctation repair. Therefore, how the aortic arches look long after the initial repair is inherent or specific to the patient. Even with stenting, the aortic arch will still harbor patient-specific shapes. In a way, in these patients, the shape analysis uncovered nonmodifiable features of the aortic arch that are associated with worse left ventricular performance, independently of hemodynamic important residual lesion. 